\title{
Avances de Proyecto Carbonización de Biomasa| Aprovechamiento de residuos agrícolas para el mejoramiento de las propiedades físico-químicas del suelo en áreas de cultivo
}

\author{
James, Arthur \\ Iniciativa de Integración de Tecnologías para el Desarrollo de Soluciones Ingenieriles (I²TEDSI), Facultad \\ de Ingeniería Mecánica, Universidad Tecnológica de Panamá \\ Ciudad de Panamá, Panamá \\ Sistema Nacional de Investigación (SNI) \\ Secretaria Nacional de Ciencia, Tecnología e Innovación (SENACYT) \\ arthur.james@utp.ac.pa \\ Ramírez, Joisleen \\ Iniciativa de Integración de Tecnologías para el Desarrollo de Soluciones Ingenieriles (I²TEDSI), Facultad \\ de Ingeniería Mecánica, Universidad Tecnológica de Panamá \\ Los Santos, Panamá \\ joisleen.ramirez@utp.ac.pa \\ Cedeño, Maritza \\ Facultad de Ingeniería Industrial, Universidad Tecnológica de Panamá Centro Regional de Azuero \\ Los Santos, Panamá \\ martiza.cedeño@utp.ac.pa \\ Marín, Nacarí \\ Iniciativa de Integración de Tecnologías para el Desarrollo de Soluciones Ingenieriles (I²TEDSI), Facultad \\ de Ingeniería Mecánica, Universidad Tecnológica de Panamá \\ Los Santos, Panamá \\ nacari.marin@utp.ac.pa \\ Serrano, Eny \\ Departamento de Hidráulica, Sanitaria y Ciencias Ambientales, Universidad Tecnológica de Panamá \\ Centro Regional de Azuero \\ Los Santos, Panamá \\ eny.serrano@utp.ac.pa

\section{Álvarez, Humberto} \\ Facultad de Ingeniería Industrial, Universidad Tecnológica de Panamá \\ Ciudad de Panamá, Panamá \\ humberto.alvarez@utp.ac.pa
}




\section{Abstract}

Organic solid waste is a raw material with the potential to produce energy and materials of medium and high-added value. Organic waste includes residual biomass from agriculture and livestock activities that can promote a circular economy and sustainability development in different areas. This project aims to carbonize agro-industrial residues for their application in soils of agricultural regions. This could help to improve the physicochemical properties of the soil. In this document, a summary of the advances in the identification of available biomass was presented. This study was centered on the Azuero region since the soils of this region have been reported to be degraded. As part of previous results, eight residues with the potential to be carbonized were identified. Surveys were applied to agro-industrial producers, and national databases were used to calculate the quantity of unused residues for specific crops. The results for rice and corn residues are presented in this document. It was identified that the rice residue in the districts of Tonosí, Pedasí, and Pocrí were approximately 52140 tonnes, 11984 tonnes, and 3727 tonnes, respectively. For corn residue, the districts of Los Santos, Pocrí, and Las Tablas generated around 14045 tons, 12406 tons, and 12,264 tons, respectively. Therefore, the amount of biomass generated in the region could represent an opportunity for its implementation in different activities, including carbonization, to improve the quality of agricultural areas.

Keywords: Biomass, soils, biochar, corn residue, rice residue.

\section{Resumen}

Los residuos sólidos orgánicos son una materia prima con potencial para producir energía y materiales de medio y alto valor agregado. Los residuos orgánicos incluyen biomasa residual de actividades agrícolas y ganaderas que pueden promover una economía circular y el desarrollo de la sostenibilidad en diferentes áreas. Este proyecto tiene como objetivo la carbonización de residuos agroindustriales para su aplicación en suelos de regiones agrícolas. Esto podría ayudar a mejorar las propiedades fisicoquímicas del suelo. En este documento se presentó un resumen de los avances en la identificación de biomasa disponible. Este estudio se centró en la región de Azuero ya que se ha informado que los suelos de esta región están degradados. Como parte de los resultados anteriores, se identificaron ocho residuos con potencial de carbonización. Se aplicaron encuestas a productores agroindustriales y se utilizaron bases de datos nacionales para calcular la cantidad de residuos no utilizados para cultivos específicos. Los resultados para residuos de arroz y maíz se presentan en este documento. Se identificó que el residuo de arroz en los distritos de Tonosí, Pedasí y Pocrí fue de aproximadamente 52140 toneladas, 11984 toneladas y 3727 toneladas, respectivamente. 
Para el residuo de maíz, los distritos de Los Santos, Pocrí y Las Tablas generaron alrededor de 14045 toneladas, 12406 toneladas y 12264 toneladas, respectivamente. Por lo tanto, la cantidad de biomasa generada en la región podría representar una oportunidad para su implementación en diferentes actividades, incluida la carbonización, para mejorar la calidad de las áreas agrícolas.

Palabras claves: Biomasa, suelos, biocarbón, residuo de maíz, residuo de arroz.

\section{INTRODUCCIÓN}

Biomasa es todo material orgánico derivado de organismos vivos, como plantas o animales, así como desechos orgánicos (lodos residuales) y residuos agroindustriales [1], [2]. Esta materia orgánica procedente de desechos agroindustriales, material residual, organismos vivos, entre otros se puede utilizar como fuente energética para la generación de calor, electricidad o la producción de sustratos orgánicos [1], [3]. La industria agrícola genera gran cantidad de residuos que podrían ser utilizados para la producción de biomateriales y energía. Sin embargo, no son aprovechados en su totalidad. Una parte de los residuos generados se emplean como alimento de animales. Mientras que, la biomasa agroindustrial restante es desechada o quemada, ya que su uso como fuente energética es poco conocida. La biomasa como materia orgánica se genera mediante el proceso de fotosíntesis. Este junto a la etapa de descomposición y transformación de la biomasa en residuos no comestibles, podrían convertir a los desechos agroindustriales en una posible fuente de contaminación ambiental. Debido a los gases que se forman en el proceso de descomposición y a la mala gestión de los desechos [4].

Los residuos agroindustriales como material orgánico pueden ser sometidos a procesos térmicos y termoquímicos, como pirólisis y gasificación, respectivamente. Esto para transformar la biomasa en biocarbón. Lo que, por las propiedades fisicoquímicas que este presenta por su naturaleza porosa, podría aumentar la capacidad de retención de agua en el suelo, mejorar la interacción entre el suelo, las plantas y los microrganismos [5]. La pirólisis ocurre en un rango de temperatura de $300{ }^{\circ} \mathrm{C}$ a $900{ }^{\circ} \mathrm{C}$ [1], [6]. El proceso de gasificación presenta rendimientos entre $5 \%$ y $10 \%$ para la conversión de biomasa a biocarbón. Estos valores son bajos, debido a que este tratamiento es mayormente utilizado para la generación de gases [1].

Actualmente, existe evidencia científica sobre el uso de biocarbón para el mejoramiento de los suelos. El biocarbón a base de residuos agroindustriales, como cascarilla de arroz aumenta los macro y microagregados en el suelo [7]. Además, tiende a aumentar la 
disponibilidad de fósforo (P), potasio (K), calcio (Ca) y magnesio (Mg) en el suelo. También, mejora la condición física de estos y aumenta la capacidad de intercambio catiónico y eleva el $\mathrm{pH}$ [8]. Los nutrientes que posee el biocarbón depende de las propiedades de la biomasa previo al proceso de carbonización. La estructura molecular de la cascarilla de arroz presenta alta estabilidad química y microbiana. Además, muestra estructura porosa lo que podría mejorar la retención de agua en el suelo [9].

En Panamá gran parte del territorio presenta suelos degradados. Se estima que el $27 \%$ del territorio presenta esta condición. Las áreas más críticas se ubican en Cerro Punta, Comarca Ngöbe Buglé, la Sabana Central Veragüense y el Arco Seco. Esta última región la comprenden las provincias de Herrera, Los Santos, Coclé, y parte de la provincia de Panamá. No obstante, es una de las regiones que presenta una alta producción agropecuaria en el país, siendo esta zona el 50.4\% del territorio que conforman estas 4 provincias [10], [11].

Además, parte de las provincias de Chiriquí, Veraguas y la zona de las costas del Pacífico (que corresponde las provincias de Herrera, Los Santos, Coclé) presentan las menores precipitaciones al año (1000 - $1300 \mathrm{~mm} / a$ ño). Estas tierras poseen características propias de lugares secos, alta saturación de aluminio y bajas proporciones de $\mathrm{Ca}, \mathrm{Mg}, \mathrm{K}$ aunado a las bajas precipitaciones dan como resultado un suelo degradado y seco [11]. Por lo que, este proyecto plantea la carbonización de residuos agroindustriales para su aplicación en suelos en áreas de cultivo y así mejorar las propiedades fisicoquímicas de este.

\section{MÉTODO}

\section{A. Encuestas}

Se evaluaron estadísticamente los residuos agroindustriales de la región de Azuero, por medio de encuestas. Estas fueron aplicadas a productores de cultivos de arroz y maíz. Este paso se realizó con el objetivo de determinar los cultivos que presenten mayor potencial, en cuanto a su producción. Y así, proponer su posible uso como mejoradores de las propiedades fisicoquímicas de los suelos. Las interrogantes de la encuesta fueron puntuales con respecto a ¿cuántas cosechas se realiza al año del cultivo en cuestión?, ¿en qué meses del año se realizan estas cosechas?, ¿qué porcentaje del residuo generado utiliza el productor? Además, se realizaron preguntas sobre ¿qué tipo de residuos genera?, si genera residuos por rechazo de producto, si utiliza un porcentaje de la biomasa generada en otra actividad, de ser cierto, ¿en qué actividades los aprovecha? En caso de que los residuos no se aprovechen en actividades varias, ¿qué hace con estos residuos? (si los venden, los queman o los trasladan a otros lugares).

Previo a la aplicación de las encuestas se realizó una investigación en el Instituto Nacional de Estadística y Censo de la Contraloría General de la Republica (INEC). Ya que la región de Azuero es una de las áreas con mayor producción agrícola y, por lo tanto, cuenta con 
gran cantidad de productores de diversos cultivos [10], [11] . Uno de los cultivos de mayor producción en esta región es el cultivo de maíz mecanizado con $97 \%$ de la producción a nivel nacional en el periodo 2018-2019 [12]. De este rubro se contabiliza un total de 408 productores. Mientras que, para el arroz se registran 184 productores [13]. La suma de todos los productores anteriores da como resultado la población heterogénea. Por lo que se utilizó la relación (2) de Murray y Larry [14].

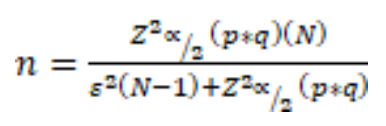

En donde $n$, es el tamaño de la muestra; a las variables $p$ y q se les asignó un valor de $50 \%$ a cada una (valor neutral cuando no hay referencia de investigaciones anteriores). El valor del error, $\varepsilon$, debido a las limitaciones del estudio se determinó con un valor de 0.12 o $12 \%$. Se utilizó un nivel de confianza de $95 \%$ para lo cual el valor de $Z$ fue de 1.96 y N se describió como tamaño de la población la cual tuvo un valor de 714 para estos cultivos. Al reemplazar en la ecuación (2), obtenemos $n=62$ para el arroz y el maíz mecanizado. Con estos valores se calculó ni, según cada estrato de la población utilizando la relación (3).

$$
\mathrm{ni}=(\mathrm{Ni} / \mathrm{N}) * \mathrm{n}
$$

En donde ni, representa la $\mathrm{n}$ de cada estrato; $\mathrm{N}$ tamaño de la población; $\mathrm{Ni}$, tamaño de cada estrato de la población y $n$, tamaño de la muestra calculado según la relación (2).

\section{B. Residuos generados por distrito}

Se realizó una estimación de los residuos generados por distritos de la provincia de Herrera y Los Santos, tomando como base los resultados obtenidos en las encuestas y la revisión bibliográfica. Para el cultivo de arroz cosechado en campo se contabilizó la cascarilla y paja de arroz. En el rubro de maíz de campo se estudió el rastrojo, la tuza y el capullo del maíz. Para la estimación de la cantidad de residuos de arroz generado por distrito se utilizaron los datos obtenidos por Morales et al., 2016 [15]. En el cual se evidencia que se genera 1.5 ton de residuos de arroz por tonelada de producto generado en campo [15]. Mientras que, en los molinos se produce $20 \%$ del residuo de cascarilla de arroz. Por otra parte, del cultivo de maíz se produce entre un $44 \%$ a $53 \%$ de residuo, lo que indica que aproximadamente, la mitad del cultivo sembrado en campo es rastrojo y lo restante granos de maíz [15]. Para este estudio se designó un porcentaje de residuo de $44 \%$ y $56 \%$ de granos cosechados en campo. Mientras que, en los molinos se genera $19.7 \%$ de residuos de pulidura, harina, hojuela y granos partidos. 


\section{RESULTADOS}

Las encuestas fueron aplicadas a 16 productores de arroz y 36 de maíz, calculado por medio de las ecuaciones (2) y (3). Estos resultados representaron el $8,70 \%$ de los productores de arroz en la región de Azuero y 8,82\% para el cultivo de maíz. Como resultados de estas encuestas se obtuvieron datos de referencia para la designación de los posibles cultivos a utilizar para su carbonización. Algunos datos fueron, porcentaje de productores que utilizan el residuo $68,75 \%$ para el cultivo de arroz y $72,22 \%$ para maíz. Mientras que, el porcentaje restante son los productores que si utilizan el residuo para diferentes actividades, como alimento del ganado, cobertura para mantener la humedad en el suelo y como control para impedir el crecimiento de la maleza para la siguiente siembra [16]. Otro índice importante fue el porcentaje de productores que generan residuos por rechazo de producto en campo, $19 \%$ para el arroz y 0\% para el maíz.

En la tabla 1, se observa que el distrito de Tonosí perteneciente a la provincia de Los Santos presentó la mayor generación de residuos de arroz, aproximadamente, produce 52140 tonnes de desechos. De manera similar, en la provincia de Herrera el distrito de Ocú generó la mayor cantidad de residuos con 3402 tonnes. Se puede observar que, la provincia de Los Santos mostró mayor generación de residuos de Arroz que la provincia de Herrera.

Tabla 1. Estimación de residuo de arroz en las provincias de herrera y los santos, según distrito. Período/2018 - 2019.

Fuente: [15] y propia.

\begin{tabular}{|c|c|c|c|c|c|c|c|c|c|}
\hline Provincias & \multicolumn{3}{|c|}{ Herrera } & \multicolumn{5}{c|}{ Los Santos } \\
\hline Distritos & Ocú & $\begin{array}{c}\text { Santa } \\
\text { María }\end{array}$ & Parita & $\begin{array}{c}\text { Las } \\
\text { Tablas }\end{array}$ & $\begin{array}{c}\text { Los } \\
\text { Santos }\end{array}$ & $\begin{array}{c}\text { Tres Que- } \\
\text { bradas }\end{array}$ & Pedasí & Pocrí & Tonosí \\
\hline $\begin{array}{c}\text { Total de residuo por } \\
\text { distrito (tonnes) }\end{array}$ & 3402 & 1209 & 562 & 1227 & 2549 & 355 & 11984 & 3727 & 52140 \\
\hline
\end{tabular}

El cultivo de arroz requiere un suelo con $\mathrm{pH}$ entre 5,0 y 7,0, ya que menor que 5,0 presenta exceso de $\mathrm{Al}, \mathrm{Fe}, \mathrm{Mn}$ y deficiencia de $\mathrm{P}, \mathrm{K}$. El cultivo de arroz requiere un contenido bajo de $\mathrm{Al}, \mathrm{Na}$, y otras sales. Además, de una presencia normal de Fe y $\mathrm{Mn}$ [17]. En la provincia de Los Santos el $36 \%$ del territorio presenta un pH entre 5,6 y 5,9. Mientras que, Herrera el $51 \%$ del territorio muestra un $\mathrm{pH}<5,0$. Además, en esta provincia se encontró que el $36 \%$ de su territorio muestra una saturación de aluminio $>25 \%$. Mientras que, en Los Santos solo el $11 \%$ de los suelos presenta saturación de aluminio [11]. Por lo anterior se infiere que, la provincia de Los Santos presenta mayor siembra de arroz en comparación con la provincia de Herrera, debido a las propiedades que este suelo presenta y que son compatibles con los requerimientos para la siembra de arroz.

En la tabla 2, se observa que los distritos de Los Santos, Pocrí y Las Tablas presentaron la mayor generación de residuos de maíz con 14045 tonnes, 12406 tonnes y 12264 
tonnes, respectivamente. Mientras que, en la provincia de Herrera el distrito de Parita y Chitré presentaron la mayor generación de residuos con 4156 tonnes y 1473 tonnes, respectivamente. Se puede observar que, de la Región de Azuero la provincia de Los Santos muestra la mayor generación de residuos de maíz.

Tabla 2. Estimación de residuo de maíz en las provincias de herrera y los santos, según distrito.

Período/2018 - 2019. Fuente: [15] y propia.

\begin{tabular}{|c|c|c|c|c|c|c|c|c|c|c|c|c|c|}
\hline Provincia & \multicolumn{9}{|c|}{ Herrera } & \multicolumn{7}{c|}{ Los Santos } \\
\hline Distritos & Chitré & Pesé & Ocú & $\begin{array}{c}\text { Las } \\
\text { Minas }\end{array}$ & $\begin{array}{c}\text { Santa } \\
\text { María }\end{array}$ & Parita & $\begin{array}{c}\text { Las } \\
\text { Tablas }\end{array}$ & Guararé & $\begin{array}{c}\text { Los } \\
\text { Santos }\end{array}$ & $\begin{array}{c}\text { Maca- } \\
\text { racas }\end{array}$ & Pedasí & Pocrí & Tonosí \\
\hline $\begin{array}{c}\text { Total de } \\
\text { residuo } \\
\text { por } \\
\text { distrito } \\
\text { (tonnes) }\end{array}$ & 1473 & 815 & 247 & 71 & 562 & 4156 & 12264 & 8746 & 14045 & 854 & 8302 & 12406 & 349 \\
\hline
\end{tabular}

El cultivo de maíz tiende a ser cultivados en diferentes tipos de suelos. Sin embargo, presenta dificultades de crecimiento en suelos arcillosos, ya que son excesivamente pesados y en suelos arenosos debido a que son muy sueltos [18]. El cultivo de maíz crece en suelos con pH entre 5,5 y 7,8 [19], no obstante el pH adecuado para un crecimiento óptimo corresponde a un $\mathrm{pH}$ entre 6,0 y 7,0 [18]. Esto se debe a que suelos con $\mathrm{pH}$ superior a 7,0 muestran déficit en $\mathrm{Fe}, \mathrm{Mn}$ y $\mathrm{Zn}$. Mientras que, $\mathrm{pH}$ inferiores a 5,5 presentan dificultades como toxicidad por Al y $\mathrm{Mn}$ y una carencia de $\mathrm{Mg}$ y $\mathrm{P}$ [19]. Por lo que, se infiere que la provincia de Los Santos presentó mayor generación de residuos de maíz, ya que los suelos de esta provincia presentan características similares a los requerimientos del cultivo para un desarrollo óptimo. Esto comparado con los suelos de la provincia de Herrera, que muestra un $\mathrm{pH}$ inferior a 5,0 lo que podría indicar una mayor saturación de Al en sus suelos [11].

\section{CONCLUSIONES}

Con este avance en el Proyecto de Carbonización de Biomasa se logró demostrar que en la región de Azuero se producen diversos cultivos de los cuales se generan toneladas de residuos. Los residuos del cultivo de maíz son mayormente utilizados por los productores en comparación con los residuos de arroz. No obstante, se genera mayor cantidad de residuos de maíz que del arroz. Sin embargo, este último es poco utilizado en la región, en comparación con la biomasa del cultivo de maíz. Debido a estos resultados, se concluye que ambos cultivos, en cuanto a la generación de residuos, presentan potencial para ser carbonizados y posteriormente ser utilizados como posibles mejoradores de las propiedades fisicoquímicas del suelo. 


\section{Referencias}

[1] J. S. Cha et al., "Production and utilization of biochar: A review," Journal of Industrial and Engineering Chemistry, vol. 40, pp. 1-15, 2016.

[2] S. Clarke, "Biomass Burn Characteristics," Ministry of Agriculture, Food and Rural Affairs, no. 11, pp. 1-7, 2017.

[3] The Need Proyect, "Biomass," 2018.

[4] K. Cury R, Y. Aguas M, A. Martinez M, R. Olivero V, and L. Chams Ch, "Residuos agroindustriales su impacto, manejo y aprovechamiento," Revista Colombiana de Ciencia Animal - RECIA, vol. 9, no. S, p. 122, 2017.

[5] S. Abel, A. Peters, S. Trinks, H. Schonsky, M. Facklam, and G. Wessolek, "Impact of biochar and hydrochar addition on water retention and water repellency of sandy soil," Geoderma, vol. 202-203, pp. 183-191, 2013.

[6] J. Carvajal Muñoz and A. Mera Benavides, "Biological fertilization: state of the art techniques for a sustainable agricultural development," Producción + Limpia, vol. 5, no. 2, pp. 77-96, 2010.

[7] S. G. Lu, F. F. Sun, and Y. T. Zong, "Effect of rice husk biochar and coal fly ash on some physical properties of expansive clayey soil (Vertisol)," Catena, vol. 114, pp. 37-44, 2014.

[8] X. Zhao, J. Wang, S. Wang, and G. Xing, "Successive straw biochar application as a strategy to sequester carbon and improve fertility: A pot experiment with two rice/wheat rotations in paddy soil," Plant and Soil, vol. 378, no. 1-2, pp. 279-294, 2014.

[9] C. J. Atkinson, J. D. Fitzgerald, and N. A. Hipps, "Potential mechanisms for achieving agricultural benefits from biochar application to temperate soils: A review," Plant and Soil, vol. 337, no. 1, pp. 1-18, 2010.

[10] Miambiente, Atlas De Las Tierras Secas y Degradadas De Panamá. 2009.

[11] J. E. Villarreal, I. Ramos, J. Villalaz, and A. Santo, "Clasificación taxonómica y caracterización físicoquímica de los suelos de la región de Azuero-Panamá," pp. 1-7, 2017.

[12] Ministerio de Desarrollo Agropecuario, “Información general, año 2018-2019," 2019.

[13] Instituto Nacional de Estadísitica y Censo, "Volumen II. Características de los Productores y la Exportaciones Agropecuarias," 2011. [Online]. Available: https://www.inec.gob.pa/publicaciones/ Default3.aspx?ID_PUBLICACION=443\&ID_CATEGORIA=15\&ID_SUBCATEGORIA=60.

[14] S. Murray R. and S. Larry J., Estadística, 4th ed. Mexico, D.F.: McGraw-Hill, 2009.

[15] R. Morales, L. Agudo, and J. Espinosa, "Disponibilidad De Residuos De Cosecha De Arroz Y Maíz Para La Producción De Etanol," no. 24, pp. 83-92, 2016.

[16] Proyecto Especial para la Seguridad Alimentaria (PESA) Honduras, "Manejo de humedad del suelo en zonas secas," Manejo de sistemas agroforestales, 2005.

[17] A. Frye, J. E. Baquero, J. E. Carvajal, and M. J. Villota, "Suelos y fertilización en el cultivo de arroz en Colombia," Universidad de Tolima, 1991.

[18] A. E. Cigarruista, "EFECTO DEL DÉFICIT HÍDRICO Y TEMPERATURA AMBIENTAL SOBRE EL CULTIVO DE MAÍZ EN EL EJIDO DE LOS SANTOS," Universidad de Panamá, 2018.

[19] H. D. Flores, “Guía Técnica El cultivo de maíz," 2012. 


\section{Autorización y Licencia CC}

Los autores autorizan a APANAC XVIII a publicar el artículo en las actas de la conferencia en Acceso Abierto (Open Access) en diversos formatos digitales (PDF, HTML, EPUB) e integrarlos en diversas plataformas online como repositorios y bases de datos bajo la licencia, Attribution-NonCommercialShareAlike 4.0 International (CC BY-NC-SA 4.0) https://creativecommons.org/licenses/by-nc-sa/4.0/.

Ni APANAC XVIII ni los editores son responsables ni del contenido ni de las implicaciones de lo expresado en el artículo. 